Discussion Paper No. 964

\title{
STRATEGY-PROOF \\ PROBABILISTIC MECHANISMS \\ FOR \\ PUBLIC DECISION WITH MONEY
}

\author{
Kazuhiko Hashimoto \\ Kohei Shiozawa
}

March. 2016

The Institute of Social and Economic Research

Osaka University

6-1 Mihogaoka, Ibaraki, Osaka 567-0047, Japan 


\title{
Strategy-Proof Probabilistic Mechanisms for Public Decision with Money
}

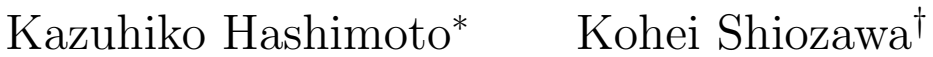

March 22, 2016

\begin{abstract}
We study strategy-proof probabilistic mechanisms in a binary public decision model when monetary transfers are allowed. We consider not only the pivotal mechanism, the majority voting mechanism, the random serial dictatorship mechanism, and the unanimity mechanism, but also the random chair pivotal mechanism (Faltings 2005), which is a probabilistic variant of the pivotal mechanism.

We first show that the random chair pivotal mechanism, the majority voting mechanism, the random serial dictatorship mechanism, and the unanimity mechanism are second-best efficient.

Next, we calculate the expected welfare of the mechanisms by the Monte Carlo method, where each agent's valuation is independently, identically, and uniformly (or normally) distributed. These calculations exhibit that the random chair pivotal mechanism is more efficient than the other mechanisms. We also show that in large economies, the random chair pivotal mechanism is efficient, while the other mechanisms might be highly inefficient.

Finally, we characterize the random chair pivotal mechanism with strategy-proofness, budget-balance, equal treatment of equals, and decision-robustness.
\end{abstract}

Keywords: Strategy-proofness; Budget-balance; Second-best efficiency; Public decision; Pivotal mechanism; Random chair pivotal mechanism.

JEL codes: D71; H41.

${ }^{*}$ Institute of Social and Economic Research, Osaka University, 6-1 Mihogaoka, Ibaraki, Osaka 567-0047, JAPAN; Email: k-hashimoto@iser.osaka-u.ac.jp

${ }^{\dagger}$ Graduate School of Economics, Osaka University, Machikaneyama, Toyonaka, Osaka 560-0043, JAPAN; Email: nge013sk@student.econ.osaka-u.ac.jp 


\section{Introduction}

We study a mechanism design problem in a binary public decision model when monetary transfers are allowed. Each agent has a quasi-linear preference. A (direct) mechanism determines a public decision and monetary transfers depending on agents' preferences. We focus on mechanisms satisfying strategy-proofness, which requires that it be a dominant strategy for any agent to report his true preference. ${ }^{1}$

The pivotal mechanism (Clarke 1971) is the most well-known mechanism ${ }^{2}$ on this model. It satisfies not only strategy-proofness but also decisionefficiency. Decision-efficiency requires that the mechanism select a public decision that maximizes the aggregate valuation. Moreover, it is the only mechanism satisfying strategy-proofness, decision-efficiency, and some welfare lower bound property (Moulin 1986).

Although the pivotal mechanism has the special features, it also has several drawbacks. ${ }^{3}$ A well-known drawback is that it does not satisfy budgetbalance. Budget-balance requires that the monetary transfers be closed among the agents. On the quasi-linear environments, decision-efficiency and budget-balance are equivalent to Pareto-efficiency. Hence, the pivotal mechanism is not Pareto-efficient. However, this drawback is not particular to the pivotal mechanism, because there exists no mechanism satisfying strategyproofness and Pareto-efficiency (Green and Laffont 1977). ${ }^{4}$ Furthermore, this negative result is valid even when we admit probabilistic mechanisms (Holmström 1979). Thus, we cannot obtain the first-best strategy-proof mechanism. $^{5}$

Then, our next aim is to find good second-best efficient mechanisms among strategy-proof ones. Second-best efficiency requires that the mechanism be on the Pareto-frontier among mechanisms. ${ }^{6}$ As candidates for such mechanisms, we consider well-known ones; the majority voting mechanism,

\footnotetext{
${ }^{1}$ Carbajal et al. (2013) have comprehensively analyzed strategy-proof mechanisms. See also Mishra and Sen (2012) and Marchant and Mishra (2015).

${ }^{2}$ This is sometimes called VCG mechanism (Vickrey 1961; Clarke 1971; Groves 1973).

${ }^{3}$ They have been mentioned in Groves and Ledyard (1977) and discussed in Green and Laffont (1979).

${ }^{4}$ On continuous public decision models with very restricted domains, strategy-proofness and Pareto-efficiency are sometimes compatible (Groves and Loeb 1975; Green and Laffont 1979; Laffont and Maskin 1980; Tian 1996; Liu and Tian 1999).

${ }^{5}$ Hence, Moulin (1994), Serizawa (1996, 1999), and Ohseto (2000) have researched strategy-proof mechanisms satisfying budget-balance instead of decision-efficiency. See also Bailey (1997).

${ }^{6}$ Zhou (2007) has analyzed a second-best efficient mechanism with regard to expected social welfare.
} 
the random serial dictatorship mechanism, and the unanimity mechanism. We also consider a mechanism that is a probabilistic variant of the pivotal mechanism, called the random chair pivotal mechanism (Faltings 2005). First, it selects randomly one agent called "chair." Second, the pivotal mechanism among the agents except the chair determines the public decision and their transfers. Finally, the chair obtains the surplus derived in the previous step. $^{7}$

We first show that the random chair pivotal mechanism, the majority voting mechanism, the random serial dictatorship mechanism, and the unanimity mechanism are second-best efficient. These results say that there is no Pareto-domination among them. Hence, we next calculate the expected welfare of the mechanisms by the Monte Carlo method, where each agent's valuation is independently, identically, and uniformly (or normally) distributed. These calculations exhibit that the random chair pivotal mechanism is more efficient than the other mechanisms. We also show that in large economies, the random chair pivotal mechanism is efficient, while the other mechanisms might be highly inefficient. 8 Thus, the random chair pivotal mechanism is superior in terms of efficiency. Finally, we characterize the random chair pivotal mechanism with strategy-proofness, budget-balance, equal treatment of equals, and decision-robustness. Equal treatment of equals requires that the agents who have the same preference get the same assignment. Decisionrobustness requires that we respect for only the support for the public decision that is robust in some sense. Therefore, the random chair pivotal mechanism is an excellent strategy-proof mechanism.

The paper is organized as follows. In Section 2, we set up the model. In Section 3, we introduce the mechanisms. In Section 4, we state our results. In Section 5, we provide the proofs.

\section{Model}

Let $N=\{1,2, \ldots, n\}$ be the set of agents, where we assume $n \geq 3$. We consider an environment with a binary public decision on $\{0,1\}$ and one divisible good called money. The binary public decision can be made probabilistically.

Each agent $i \in N$ has a preference over the set of pairs consisting of a probability $s \in[0,1]$ that the society selects the public decision 1 and a monetary transfer $t_{i} \in \mathbb{R}$ that he receives. We assume that this preference is represented by a utility function $u_{i}\left(s, t_{i} ; v_{i}\right)=s v_{i}+t_{i}$ for some $v_{i} \in V \equiv \mathbb{R}$.

\footnotetext{
${ }^{7}$ See also Guo et al. (2011).

${ }^{8}$ Green et al. (1976), Green and Laffont (1979), Rob (1982), and Mitsui (1983) have studied the budget surplus of the pivotal mechanism in large economies.
} 
Since a preference is identified by $v_{i}$, we regard $v_{i}$ and $V$ as the preference and the set of preferences, respectively. We call a list $v \equiv\left(v_{i}\right)_{i \in N} \in V^{n}$ a preference profile.

The set of feasible allocations is

$$
Z=\left\{\left(s, t_{1}, \ldots, t_{n}\right) \in[0,1] \times \mathbb{R}^{n}: \sum_{i \in N} t_{i} \leq 0\right\} .
$$

A mechanism is a function $f: V^{n} \rightarrow Z$. Given a mechanism $f$ and a preference profile $v \in V^{n}$, we denote $f(v) \equiv(s(v), t(v)) \equiv\left(s(v), t_{1}(v), \ldots, t_{n}(v)\right)$, where $s(v)$ and $t_{i}(v)$ mean the probability and agent $i$ 's transfer at $v$, respectively. For any $v \in V^{n}$ and $N^{\prime} \subseteq N$, let $v_{N^{\prime}} \in V^{\# N^{\prime}}$ and $v_{-N^{\prime}} \in V^{\# N \backslash N^{\prime}}$ denote $\left(v_{j}\right)_{j \in N^{\prime}}$ and $\left(v_{j}\right)_{j \notin N^{\prime}}$, respectively.

We define the basic properties. First, strategy-proofness says that it is a dominant strategy for any agent to report his true preference.

Definition 1. A mechanism $f$ satisfies strategy-proofness (SP) if for any $v \in V^{n}$, any $i \in N$, and any $v_{i}^{\prime} \in V$, it holds that

$$
s(v) v_{i}+t_{i}(v) \geq s\left(v_{i}^{\prime}, v_{-i}\right) v_{i}+t_{i}\left(v_{i}^{\prime}, v_{-i}\right) .
$$

Next, second-best efficiency says that the mechanism is on the Paretofrontier among strategy-proof mechanisms.

Definition 2. A strategy-proof mechanism $f^{*}$ is second-best efficient if there does not exist another strategy-proof mechanism $f$ such that for any $v \in V^{n}$ and any $i \in N$,

$$
s(v) v_{i}+t_{i}(v) \geq s^{*}(v) v_{i}+t_{i}^{*}(v),
$$

and for some $v \in V^{n}$ and some $j \in N$,

$$
s(v) v_{j}+t_{j}(v)>s^{*}(v) v_{j}+t_{j}^{*}(v) .
$$

\section{Mechanisms}

\subsection{Pivotal Mechanism}

We introduce the pivotal mechanism (Clarke 1971). It selects the public decision that maximizes the aggregate valuation of agents. Each agent pays the amount of money corresponding to the externality that he is imposing on the other agents. When there is a tie on the public decision, the tie is broken arbitrarily. Hence, to be precise, the pivotal mechanism is not unique. 
However, we treat the pivotal mechanism as being unique because all of them are welfare equivalent.

To define it formally, we need some notation. For any $v \in V^{n}$, define $d(v)$ as follows:

$$
d(v) \equiv \begin{cases}0 & \text { if } \sum_{k \in N} v_{k}<0 \\ y & \text { if } \sum_{k \in N} v_{k}=0 \\ 1 & \text { if } \sum_{k \in N} v_{k}>0\end{cases}
$$

where $y$ is an arbitrary value in $\{0,1\}$ and might depend on $v$.

For any $v \in V^{n}$ and any $i \in N$, define $d^{-i}(v)$ as follows: ${ }^{9}$

$$
d^{-i}(v) \equiv \begin{cases}0 & \text { if } \sum_{k \neq i} v_{k}<0 \\ 0 & \text { if } \sum_{k \neq i} v_{k}=0 \text { and } v_{i}<0 \\ y & \text { if } \sum_{k \neq i} v_{k}=0 \text { and } v_{i}=0 \\ 1 & \text { if } \sum_{k \neq i} v_{k}=0 \text { and } v_{i}>0 \\ 1 & \text { if } \sum_{k \neq i} v_{k}>0\end{cases}
$$

where $y$ is an arbitrary value in $[0,1]$ and might depend on $v$.

Definition 3. The pivotal mechanism $f^{P V}=\left(s^{P V}, t^{P V}\right)$ is defined as follows: For any $v \in V^{n}$,

$$
s^{P V}(v) \equiv d(v),
$$

and for any $i \in N$,

$$
t_{i}^{P V}(v) \equiv \sum_{k \neq i} v_{k} d(v)-\sum_{k \neq i} v_{k} d^{-i}(v)
$$

\subsection{Random Chair Pivotal Mechanism}

We introduce the random chair pivotal mechanism (Faltings 2005), which is one variant of the pivotal mechanism. First, one agent called chair is selected randomly. Next, the pivotal mechanism among the agents except the chair determines the public decision and their transfers, and the chair obtains the surplus. When there is a tie on the public decision among the agents except the chair, the chair breaks the tie. When it is also a tie for the chair, the tie is broken arbitrarily. Hence, to be precise, the random chair pivotal mechanism is not unique. However, we treat the random chair pivotal mechanism as being unique, because all of them are welfare equivalent.

\footnotetext{
${ }^{9}$ For the pivotal mechanism, $d^{-i}(v)$ is arbitrary when $\sum_{k \neq i} v_{k}=0$. This specification is to define the next mechanism.
} 
To define it formally, we need some notation. Note that we can regard $d^{-j}(v)$ as the public decision determined by the pivotal mechanism among the agents except the chair $j$. For any $v \in V^{n}$ and any $i, j \in N$ such that $i \neq j$, define $d^{-i j}(v)$ as follows:

$$
d^{-i j}(v) \equiv \begin{cases}0 & \text { if } \sum_{k \neq i, j} v_{k}<0 \\ y & \text { if } \sum_{k \neq i, j} v_{k}=0 \\ 1 & \text { if } \sum_{k \neq i, j} v_{k}>0\end{cases}
$$

where $y$ is an arbitrary value. ${ }^{10}$

For any $v \in V^{n}$ and any $i, j \in N$ such that $i \neq j$, define $p_{i}^{-j}(v)$ as follows:

$$
p_{i}^{-j}(v) \equiv \sum_{k \neq i, j} v_{k} d^{-j}(v)-\sum_{k \neq i, j} v_{k} d^{-i j}(v) .
$$

Hence, $p_{i}^{-j}(v)$ is agent $i$ 's transfer of the pivotal mechanism among the agents except the chair $j$. The chair $j$ obtains this surplus $-p_{i}^{-j}(v)$ from each agent $i \neq j$.

Definition 4. The random chair pivotal mechanism $f^{R C}=\left(s^{R C}, t^{R C}\right)$ is defined as follows. For any $v \in V^{n}$,

$$
s^{R C}(v) \equiv \frac{1}{n} \sum_{i \in N} d^{-i}(v),
$$

and for any $i \in N$,

$$
t_{i}^{R C}(v) \equiv \frac{1}{n}\left[-\sum_{j \neq i} p_{j}^{-i}(v)+\sum_{j \neq i} p_{i}^{-j}(v)\right] .
$$

Example 1. Consider the three-agent case. Let $v=(3,2,-4)$. Then, we obtain that

$$
\begin{aligned}
& d^{-1}(v)=0 \\
& d^{-2}(v)=0 \\
& d^{-3}(v)=1
\end{aligned}
$$

We also obtain that

$$
d^{-12}(v)=d^{-21}(v)=0,
$$

\footnotetext{
${ }^{10}$ We need $d^{-i j}(v)$ to define $p_{i}^{-j}(v)$. In the definition of $p_{i}^{-j}(v)$, when $\sum_{k \neq i, j} v_{k}=0$, we have $\sum_{k \neq i, j} v_{k} d^{-i j}(v)=0$ regardless of value of $d^{-i j}(v)$. Hence, we allow $d^{-i j}(v)$ to take an arbitrary value.
} 


$$
\begin{aligned}
& d^{-13}(v)=d^{-31}(v)=1 \\
& d^{-23}(v)=d^{-32}(v)=1 .
\end{aligned}
$$

Hence, it follows that

$$
\begin{aligned}
& p_{2}^{-1}(v)=0 \text { and } p_{3}^{-1}(v)=-2, \\
& p_{1}^{-2}(v)=0 \text { and } p_{3}^{-2}(v)=-3, \\
& p_{1}^{-3}(v)=0 \text { and } p_{2}^{-3}(v)=0 .
\end{aligned}
$$

Thus, the allocation of the random chair pivotal mechanism at $v$ is

$$
s^{R C}(v)=\frac{1}{3} \text { and } t^{R C}(v)=\left(\frac{2}{3}, \frac{3}{3},-\frac{5}{3}\right) .
$$

\subsection{Other Mechanisms}

We introduce the other mechanisms that are well-known in mechanism design or social choice theory. These mechanisms always make no transfer among agents.

First, we introduce the majority voting mechanism. It selects the public decision that is supported by the majority.

Definition 5. The majority voting mechanism $f^{M V}=\left(s^{M V}, t^{M V}\right)$ is defined as follows. For any $v \in V^{n}$,

$$
s^{M V}(v) \equiv \begin{cases}0 & \text { if } \#\left\{i \in N: v_{i}>0\right\}<\#\left\{i \in N: v_{i}<0\right\} \\ \frac{1}{2} & \text { if } \#\left\{i \in N: v_{i}>0\right\}=\#\left\{i \in N: v_{i}<0\right\} \\ 1 & \text { if } \#\left\{i \in N: v_{i}>0\right\}>\#\left\{i \in N: v_{i}<0\right\}\end{cases}
$$

and for any $i \in N$,

$$
t_{i}^{M V}(v) \equiv 0
$$

Next, we introduce the random serial dictatorship mechanism. The public decision selected by this mechanism is determined as follows: We randomly fix a linear ordering on $N$. The first priority agent chooses the public decision according to his favor. If the first priority agent is indifferent between $\{0,1\}$, then the second priority agent chooses the public decision according to her favor, and so on. We represent this mechanism simply as follows.

Definition 6. The random serial dictatorship mechanism $f^{R D}=\left(s^{R D}, t^{R D}\right)$ is defined as follows. For any $v \in V^{n}$,

$$
s^{R D}(v) \equiv \begin{cases}\frac{\#\left\{i \in N: v_{i}>0\right\}}{\#\left\{i \in N: v_{i} \neq 0\right\}} & \text { if } \#\left\{i \in N: v_{i} \neq 0\right\} \neq 0, \\ \frac{1}{2} & \text { if } \#\left\{i \in N: v_{i} \neq 0\right\}=0\end{cases}
$$


and for any $i \in N$,

$$
t_{i}^{R D}(v) \equiv 0
$$

Finally, we introduce the unanimity mechanism. It selects the public decision that is unanimously supported. When there is no such public decision, it selects 0 or 1 with an equal probability for both.

Definition 7. The unanimity mechanism $f^{U N}=\left(s^{U N}, t^{U N}\right)$ is defined as follows. For any $v \in V^{n}$,

$$
s^{U N}(v) \equiv \begin{cases}0 & \text { if } \#\left\{i \in N: v_{i} \leq 0\right\}=n \text { and } \#\left\{i \in N: v_{i}<0\right\} \neq 0, \\ 1 & \text { if } \#\left\{i \in N: v_{i} \geq 0\right\}=n \text { and } \#\left\{i \in N: v_{i}>0\right\} \neq 0, \\ \frac{1}{2} \quad \text { otherwise }\end{cases}
$$

and for any $i \in N$,

$$
t_{i}^{U N}(v) \equiv 0
$$

\section{Results}

We state the results. All the proofs are provided in the final section.

\subsection{Second-Best Efficiency}

The first result says that the random chair pivotal mechanism is on the Pareto-frontier among strategy-proof mechanisms.

Theorem 1. The random chair pivotal mechanism is second-best efficient.

To establish that the majority voting mechanism, the random serial dictatorship mechanism, and the unanimity mechanism are second-best efficient, we show the following proposition.

Proposition 1. Let $f^{*}=\left(s^{*}, t^{*}\right)$ be a strategy-proof mechanism satisfying the following conditions:

(i) for any $v \in V^{n}$ and any $i \in N$,

$$
t_{i}^{*}(v)=0,
$$

(ii) for any $v \in V^{n}$ such that for any $i \in N, v_{i} \geq 0$ and that for some $i \in N$, $v_{i}>0$,

$$
s^{*}(v)=1,
$$

(iii) for any $v \in V^{n}$ such that for any $i \in N, v_{i} \leq 0$ and that for some $i \in N$, $v_{i}<0$,

$$
s^{*}(v)=0 .
$$

Then, $f^{*}$ is second-best efficient. 
Since three mechanisms satisfy the conditions (i), (ii), and (iii), we have the following corollaries.

Corollary 1. The majority voting mechanism is second-best efficient.

Corollary 2. The random serial dictatorship mechanism is second-best efficient.

Corollary 3. The unanimity mechanism is second-best efficient.

Remark 1. It is an open question whether the pivotal mechanism is secondbest efficient.

\subsection{Expected Welfare}

Since the random chair pivotal mechanism, the majority voting mechanism, the random serial dictatorship mechanism, and the unanimity mechanism are second-best efficient, there is no Pareto-domination among them. Thus, we next calculate the expected welfare of mechanisms by the Monte Carlo method and compare the mechanisms. Let $g(\cdot)$ denote a probability density function for valuation profile $v \in V^{n}$.

Definition 8. Given a mechanism $f=(s, t)$, the expected welfare of $f$ is defined as follows:

$$
E\left[\sum_{i \in N} u_{i}\left(s(v), t_{i}(v) ; v_{i}\right)\right] \equiv \int_{v \in V^{n}} \sum_{i \in N} u_{i}\left(s(v), t_{i}(v) ; v_{i}\right) g(v) d v .
$$

Let $f^{P E}$ be a Pareto-efficient mechanism, that is, a first-best efficient one. ${ }^{11}$ In Figure 1, we exhibit numerical results to compare the expected welfare of the mechanisms, where each agent's valuation is independently, identically, and uniformly distributed on $[-1,1]$. For each mechanism $f \in$ $\left\{f^{P E}, f^{P V}, f^{R C}, f^{M V}, f^{R D}, f^{U N}\right\}$ and each number of agents $n=3, \ldots, 10$, we generated 100 estimates of the expected welfare by simple Monte Carlo integration and calculated the mean value based on those 100 estimates. $^{12}$ In Figure 1, we plot the mean values for each mechanism $f$ and each number of agents $n$. These results indicate that the random chair pivotal mechanism is more efficient than the other mechanisms. Notice that the majority voting mechanism also has good performance.

\footnotetext{
${ }^{11}$ By Holmström's (1979) theorem, any first-best mechanism does not satisfy strategyproofness.

${ }^{12}$ For each set of 100 estimates, the Kolmogorov-Smirnov (KS) test failed to reject the null hypothesis of normality at the 0.05 level and the estimated standard error is less than 0.0002. All estimates were obtained using NIntegrate, which is a function implemented in Mathematica.
} 


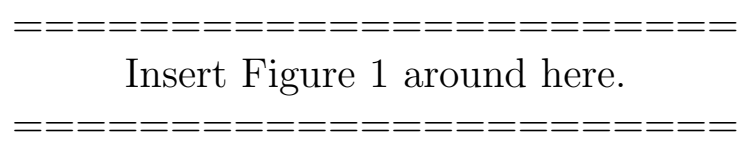

In Figure 2, we also exhibit numerical results, where each agent's valuation is independently, identically, and normally distributed with the mean 0 and the variance 1 . We estimated each of the expected welfare in the same way as the uniform distribution case. ${ }^{13}$ Figure 2 indicates that the expected welfare of each mechanism behaves in a similar manner as it does in Figure 1 .

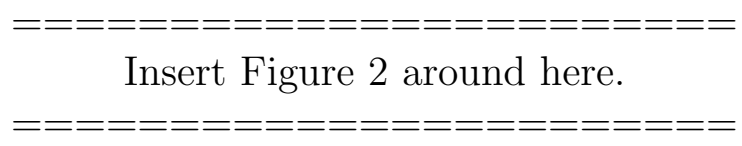

\subsection{Large Economy}

We investigate welfare of mechanisms when the economy is large. We define the welfare loss of mechanism. It is the difference between the first best welfare and the welfare of mechanism that we consider.

Definition 9. Given a mechanism $f=(s, t)$ and a preference profile $v \in V^{n}$, the welfare loss of $f$ at $v \in V^{n}$ is defined as follows:

$$
\begin{aligned}
W L(v ; f) & \equiv \max \left\{\sum_{i=1}^{n} v_{i}, 0\right\}-\left[\sum_{i=1}^{n} u_{i}\left(s(v), t_{i}(v) ; v_{i}\right)\right] \\
& =\max \left\{\sum_{i=1}^{n} v_{i}, 0\right\}-\left[s(v) \sum_{i=1}^{n} v_{i}+\sum_{i=1}^{n} t_{i}(v)\right] .
\end{aligned}
$$

We study large economies by means of replica.

Definition 10. Let a valuation profile $\left(v_{i}\right)_{i=1}^{n} \in V^{n}$. For any positive integer $r,\left(v_{i}\right)_{i=1}^{n \times r} \in V^{n \times r}$ is $r$-replica of $\left(v_{i}\right)_{i=1}^{n}$ if for any positive integer $r^{\prime}<r$ and any $i \in N$, it holds that

$$
v_{n \times r^{\prime}+i}=v_{i} .
$$

The following results say that in large economies, the random chair pivotal mechanism is efficient, while the other mechanisms might be highly inefficient.

\footnotetext{
${ }^{13}$ For each set of 100 estimates, the KS test failed to reject the null hypothesis of normality at the 0.05 level and the estimated standard error is less than 0.002 .
} 
Theorem 2. For any finite sequence of valuations $\left(v_{i}\right)_{i=1}^{n}$, the welfare loss of the random chair pivotal mechanism at $\left(v_{i}\right)_{i=1}^{n \times r}$ that is $r$-replica of $\left(v_{i}\right)_{i=1}^{n}$ converges to 0 as $r \rightarrow \infty$, that is,

$$
\lim _{r \rightarrow \infty} W L\left(\left(v_{i}\right)_{i=1}^{n \times r} ; f^{R C}\right)=0 .
$$

Proposition 2. There exists a finite sequence of valuations $\left(v_{i}\right)_{i=1}^{n}$ such that the welfare loss of the pivotal mechanism at $\left(v_{i}\right)_{i=1}^{n \times r}$ that is $r$-replica of $\left(v_{i}\right)_{i=1}^{n}$ infinitely diverges as $r \rightarrow \infty$, that is,

$$
\lim _{r \rightarrow \infty} W L\left(\left(v_{i}\right)_{i=1}^{n \times r} ; f^{P V}\right)=\infty .
$$

Proposition 3. There exists a finite sequence of valuations $\left(v_{i}\right)_{i=1}^{n}$ such that the welfare loss of the majority voting mechanism at $\left(v_{i}\right)_{i=1}^{n \times r}$ that is $r$-replica of $\left(v_{i}\right)_{i=1}^{n}$ infinitely diverges as $r \rightarrow \infty$, that is,

$$
\lim _{r \rightarrow \infty} W L\left(\left(v_{i}\right)_{i=1}^{n \times r} ; f^{M V}\right)=\infty .
$$

Proposition 4. There exists a finite sequence of valuations $\left(v_{i}\right)_{i=1}^{n}$ such that the welfare loss of the random serial dictatorship mechanism at $\left(v_{i}\right)_{i=1}^{n \times r}$ that is $r$-replica of $\left(v_{i}\right)_{i=1}^{n}$ infinitely diverges as $r \rightarrow \infty$, that is,

$$
\lim _{r \rightarrow \infty} W L\left(\left(v_{i}\right)_{i=1}^{n \times r} ; f^{R D}\right)=\infty .
$$

Proposition 5. There exists a finite sequence of valuations $\left(v_{i}\right)_{i=1}^{n}$ such that the welfare loss of the unanimity mechanism at $\left(v_{i}\right)_{i=1}^{n \times r}$ that is $r$-replica of $\left(v_{i}\right)_{i=1}^{n}$ infinitely diverges as $r \rightarrow \infty$, that is,

$$
\lim _{r \rightarrow \infty} W L\left(\left(v_{i}\right)_{i=1}^{n \times r} ; f^{U N}\right)=\infty .
$$

\subsection{Characterization}

We characterize the random chair pivotal mechanism. To do so, we introduce other properties.

First, budget-balance says that the transfers among the agents are closed.

Definition 11. A mechanism $f$ satisfies budget-balance (BB) if for any $v \in V^{n}$, it holds that

$$
\sum_{i \in N} t_{i}(v)=0
$$

Second, equal treatment of equals says that the agents who have the same preference get the same assignment. 
Definition 12. A mechanism $f$ satisfies equal treatment of equals (ETE) if for any $v \in V^{n}$ and any $i, j \in N$, if $v_{i}=v_{j}$, then it holds that

$$
t_{i}(v)=t_{j}(v) .
$$

Finally, we introduce decision-robustness. Let us consider the following valuation profiles:

$$
v^{\prime}=(10,10,1), v^{\prime \prime}=(10,10,-1), v^{\prime \prime \prime}=(10,10,-15) .
$$

Note that all the aggregate valuations at the three profiles are positive. Hence, public decision 1 is efficient in decision at the three profiles. At $v^{\prime}$, all agents unanimously support it. At $v^{\prime \prime}$, although agent 3 may oppose it, the society might be able to persuade agents to select it because the support for it is by a large margin or robust in the sense that even if any one agent changes his opinion to be neutral, the society still supports it. At $v^{\prime \prime \prime}$, on the other hand, the support for it is by a narrow margin or not robust, that is, if agent 1 or agent 2 becomes neutral, the society does not support it. Then, can the society justify to select $s\left(v^{\prime \prime \prime}\right)=1$ ? Why, for example, is $s\left(v^{\prime \prime \prime}\right)=\frac{1}{3}$ bad? Formally, when $\sum_{k \in N} v_{k}>0$, we say that the support for the public decision 1 is robust with agent $i \in N$ if $\sum_{k \neq i} v_{k} \geq 0$. Similarly, when $\sum_{k \in N} v_{k}<0$, we say that the support for the public decision 0 is robust with agent $i \in N$ if $\sum_{k \neq i} v_{k} \leq 0$. Decision-robustness says that we respect for only the supports for the public decision that are robust with agents.

Definition 13. A mechanism $f$ satisfies decision-robustness (DR) if for any $v \in V^{n}$ such that $\sum_{k \in N} v_{k}>0$, it holds that

$$
s(v)=\frac{1}{n} \#\left\{i \in N: \sum_{k \neq i} v_{k} \geq 0\right\},
$$

and for any $v \in V^{n}$ such that $\sum_{k \in N} v_{k}<0$, it holds that

$$
s(v)=1-\frac{1}{n} \#\left\{i \in N: \sum_{k \neq i} v_{k} \leq 0\right\} .
$$

The following result says that the random chair pivotal mechanism is the only mechanism satisfying desirable properties.

Theorem 3. A mechanism satisfies strategy-proofness, budget-balance, equal treatment of equals, and decision-robustness if and only if it is the random chair pivotal mechanism. 
We verify that none of the axioms in Theorems 3 is redundant. We exhibit mechanisms that satisfy all but one of the axioms. Let $n=3$.

Example 2 (not SP). Let $f=(s, t)$ be as follows: for any $v \in V^{3}$,

$$
s(v)=s^{R C}(v) \text { and } t(v)=(0,0,0) .
$$

This mechanism satisfies all but not strategy-proofness.

Example 3 (not BB). Let $\alpha>0$. Let $f=(s, t)$ be as follows: for any $v \in V^{3}$

$$
s(v)=s^{R C}(v) \text { and } t(v)=\left(t_{1}^{R C}(v)-\alpha, t_{2}^{R C}(v)-\alpha, t_{3}^{R C}(v)-\alpha\right) .
$$

This mechanism satisfies all but not budget-balance.

Example 4 (not ETE). Let $\alpha>0$. Let $f=(s, t)$ be as follows: for any $v \in V^{3}$

$$
s(v)=s^{R C}(v) \text { and } t(v)=\left(t_{1}^{R C}(v)-\alpha, t_{2}^{R C}(v)+\alpha, t_{3}^{R C}(v)\right) .
$$

This mechanism satisfies all but not equal treatment of equals.

Example 5 (not DR). Let $f=(s, t)$ be as follows: for any $v \in V^{3}$,

$$
s(v)=0 \text { and } t(v)=(0,0,0) .
$$

This mechanism satisfies all but not decision-robustness.

\section{Proofs}

Throughout the proofs, we use the following lemma that have been shown by Myerson (1981).

Lemma 1 (Myerson, 1981). If a mechanism $f$ satisfies strategy-proofness, then for any $i \in N$, any $v_{i}, v_{i}^{\prime} \in V$ such that $v_{i} \leq v_{i}^{\prime}$, and any $v_{-i} \in V^{n-1}$, it holds that

$$
s_{i}\left(v_{i}, v_{-i}\right) \leq s_{i}\left(v_{i}^{\prime}, v_{-i}\right)
$$

and

$$
t_{i}\left(v_{i}^{\prime}, v_{-i}\right)=t_{i}\left(v_{i}, v_{-i}\right)-s\left(v_{i}^{\prime}, v_{-i}\right) v_{i}^{\prime}+s\left(v_{i}, v_{-i}\right) v_{i}+\int_{v_{i}}^{v_{i}^{\prime}} s\left(x_{i}, v_{-i}\right) d x_{i} .
$$
lemma.

Before proving Theorem 1 and Proposition 1, we show the following 
Lemma 2. Let $f^{*}=\left(s^{*}, t^{*}\right)$ be a mechanism satisfying budget-balance. Let $f=(s, t)$ be a mechanism such that for any $v \in V^{n}$ and any $i \in N$,

$$
s(v) v_{i}+t_{i}(v) \geq s^{*}(v) v_{i}+t_{i}^{*}(v) .
$$

Then, it follows that for any $v \in V^{n}$, if $s(v)=s^{*}(v)$, then $t(v)=t^{*}(v)$.

Proof of Lemma 2. Let $v \in V^{n}$. Assume $s(v)=s^{*}(v)$. Then, (1) implies that for all $i \in N$,

$$
t_{i}(v) \geq t_{i}^{*}(v) .
$$

Suppose to the contrary that for some $j \in N$,

$$
t_{j}(v)>t_{j}^{*}(v) .
$$

Then, by $B B$ of $f^{*}$, these imply that

$$
\sum_{i=1}^{n} t_{i}(v)>\sum_{i=1}^{n} t_{i}^{*}(v)=0
$$

which contradicts the feasibility of $f$. Thus, we have $t(v)=t^{*}(v)$.

\subsection{Proof of Theorem 1}

We show that the random chair pivotal mechanism $f^{R C}=\left(s^{R C}, t^{R C}\right)$ is second-best efficient. Let $f=(s, t)$ be a strategy-proof mechanism such that for any $v \in V^{n}$ and any $i \in N$,

$$
s(v) v_{i}+t_{i}(v) \geq s^{R C}(v) v_{i}+t_{i}^{R C}(v) .
$$

We show that for any $v \in V^{n}$ and any $i \in N$,

$$
s(v) v_{i}+t_{i}(v)=s^{R C}(v) v_{i}+t_{i}^{R C}(v) .
$$

Let $v \in V^{n}$. In the following, we divide the argument into three cases.

Case 1. $\sum_{i=1}^{n} v_{i}>0$.

From Lemma 2, it is sufficient for (3) to show $s(v)=s^{R C}(v)$.

Claim 1. $s(v) \geq s^{R C}(v)$. 
Proof of Claim 1. By adding (2) for all agents, we have

$$
s(v) \sum_{i=1}^{n} v_{i}+\sum_{i=1}^{n} t_{i}(v) \geq s^{R C}(v) \sum_{i=1}^{n} v_{i}+\sum_{i=1}^{n} t_{i}^{R C}(v) .
$$

Since $\sum_{i=1}^{n} t_{i}(v) \leq 0$ and $\sum_{i=1}^{n} t_{i}^{R C}(v)=0$, this implies that

$$
\left(s(v)-s^{R C}(v)\right) \sum_{i=1}^{n} v_{i} \geq 0 .
$$

Since $\sum_{i=1}^{n} v_{i}>0$, it follows that

$$
s(v) \geq s^{R C}(v)
$$

Claim 2. $s(v)=s^{R C}(v)$.

Proof of Claim 2. We show this claim by the following induction.

1. If $s^{R C}(v)=1$, then $s(v)=s^{R C}(v)$.

2. Let $m$ be such that $1 \leq m \leq n-1$. Assume that for any $v^{\prime} \in V^{n}$ such that $\sum_{i=1}^{n} v_{i}^{\prime}>0$ and $s^{R C}\left(v^{\prime}\right)>\frac{n-m}{n}$, we have $s\left(v^{\prime}\right)=s^{R C}\left(v^{\prime}\right)$. If $s^{R C}(v)=\frac{n-m}{n}$, then $s(v)=s^{R C}(v)$.

Since, by Claim 1, we have $s(v) \geq s^{R C}(v)$, the first part is obviously valid. In the following, we show the second part. Without loss of generality, we assume that $v_{1} \geq v_{2} \geq \cdots \geq v_{n}$.

SubClaim 2-1. $\sum_{i \neq m} v_{i}<0$.

Proof of SubClaim 2-1. Suppose to the contrary that $\sum_{i \neq m} v_{i} \geq 0$. Since $s^{R C}(v)=\frac{n-m}{n}$, there exist $m$ agents such that for such agent $j$, we have ${ }^{14}$

$$
\sum_{i \neq j} v_{i}<0
$$

Then, there exists $h \in N$ such that $m<h$ and $\sum_{i \neq h} v_{i}<0$. Since $v_{m} \geq v_{h}$, it holds that

$$
0 \leq \sum_{i \neq m} v_{i}=\sum_{i \in N} v_{i}-v_{m} \leq \sum_{i \in N} v_{i}-v_{h}=\sum_{i \neq h} v_{i}<0,
$$

which is a contradiction. Hence, $\sum_{i \neq m} v_{i}<0$.

${ }^{14}$ Note that, since $\sum_{i \in N} v_{i}>0$, it does not occur that $\sum_{i \neq j} v_{i}=0$ and $v_{j} \leq 0$. 
SubClaim 2-2. $v_{m}>0$.

Proof of SubClaim 2-2. By SubClaim 2-1, we have $\sum_{i \neq m} v_{i}<0$. Since $\sum_{i \in N} v_{i}>0$, we have the desired result.

SubClaim 2-3. Let $\bar{v}_{n}=-\sum_{i \neq m, n} v_{i}$. Then, we have $\bar{v}_{n}>v_{n}$ and $s^{R C}\left(\bar{v}_{n}, v_{-n}\right)>\frac{n-m}{n}$.

Proof of SubClaim 2-3. Note that

$$
\sum_{i \neq m, n} v_{i}+\bar{v}_{n}=0
$$

By SubClaim 2-1, it holds that

$$
\sum_{i \neq m, n} v_{i}+v_{n}=\sum_{i \neq m} v_{i}<0
$$

These imply that $\bar{v}_{n}>v_{n}$.

It implies that for any $i \in N$,

$$
d^{-i}\left(\bar{v}_{n}, v_{-n}\right) \geq d^{-i}(v) .
$$

Since, by SubClaim 2-1, $\sum_{i \neq m} v_{i}<0$, we have

$$
d^{-m}(v)=0 .
$$

By SubClaim 2-2 and (4), it holds that

$$
d^{-m}\left(\bar{v}_{n}, v_{-n}\right)=1
$$

These imply that

$$
s^{R C}\left(\bar{v}_{n}, v_{-n}\right) \equiv \frac{1}{n} \sum_{i \in N} d^{-i}\left(\bar{v}_{n}, v_{-n}\right)>\frac{1}{n} \sum_{i \in N} d^{-i}(v) \equiv s^{R C}(v)=\frac{n-m}{n} .
$$

SubClaim 2-4. For any $\hat{v}_{n}<\bar{v}_{n}$, we have $s^{R C}\left(\hat{v}_{n}, v_{-n}\right) \leq \frac{n-m}{n}$.

Proof of SubClaim 2-4. Let $\hat{v}_{n}<\bar{v}_{n}$. For any $m^{\prime}<m$, since we assume $v_{m^{\prime}} \geq v_{m}$, we have

$$
\sum_{j \neq m^{\prime}, n} v_{j}+\hat{v}_{n} \leq \sum_{j \neq m, n} v_{j}+\hat{v}_{n}<\sum_{j \neq m, n} v_{j}+\bar{v}_{n}=0 .
$$


This implies that for any $m^{\prime} \leq m$,

$$
d^{-m^{\prime}}\left(\hat{v}_{n}, v_{-n}\right)=0
$$

that is, there exist at least $m$ agents such that for such agent $j$, it holds that

$$
d^{-j}\left(\hat{v}_{n}, v_{-n}\right)=0
$$

Hence, we have

$$
\frac{1}{n} \sum_{i \in N} d^{-i}\left(\hat{v}_{n}, v_{-n}\right) \leq \frac{n-m}{n},
$$

which is the desired result.

SubClaim 2-5. For any $\hat{v}_{n} \in V$ such that $v_{n} \leq \hat{v}_{n}<\bar{v}_{n}$, we have $s^{R C}\left(\hat{v}_{n}, v_{-n}\right)=s^{R C}(v)$.

Proof of SubClaim 2-5. Let $\hat{v}_{n} \in V$ be such that $v_{n} \leq \hat{v}_{n}<\bar{v}_{n}$. Remember that $s^{R C}(v)=\frac{n-m}{n}$. By SubClaim 2-4, we have $s^{R C}\left(\hat{v}_{n}, v_{-n}\right) \leq \frac{n-m}{n}$. Since $v_{n} \leq \hat{v}_{n}$, by $S P$ of $f^{R C}$ and Myerson's Lemma, it holds that

$$
\frac{n-m}{n}=s^{R C}(v) \leq s^{R C}\left(\hat{v}_{n}, v_{-n}\right) \leq \frac{n-m}{n},
$$

which is the desired result.

SubClaim 2-6. $s^{R C}\left(\bar{v}_{n}, v_{-n}\right)=s\left(\bar{v}_{n}, v_{-n}\right)$ and $t^{R C}\left(\bar{v}_{n}, v_{-n}\right)=t\left(\bar{v}_{n}, v_{-n}\right)$.

Proof of SubClaim 2-6. By SubClaim 2-3, we have $\bar{v}_{n}>v_{n}$. Hence, it follows that

$$
\sum_{i \neq n} v_{i}+\bar{v}_{n}>\sum_{i \in N} v_{i}>0
$$

By SubClaim 2-3, we have $s^{R C}\left(\bar{v}_{n}, v_{-n}\right)>\frac{n-m}{n}$. Thus, by the induction hypothesis, it holds that

$$
s^{R C}\left(\bar{v}_{n}, v_{-n}\right)=s\left(\bar{v}_{n}, v_{-n}\right) .
$$

Then, by Lemma 2, it follows that

$$
t^{R C}\left(\bar{v}_{n}, v_{-n}\right)=t\left(\bar{v}_{n}, v_{-n}\right) .
$$

SubClaim 2-7. $s^{R C}(v)=s(v)$. 
Proof of SubClaim 2-7. By SubClaim 2-6 and SP of $f$, we have

$$
\begin{aligned}
s^{R C}\left(\bar{v}_{n}, v_{-n}\right) \bar{v}_{n}+t_{n}^{R C}\left(\bar{v}_{n}, v_{-n}\right) & =s\left(\bar{v}_{n}, v_{-n}\right) \bar{v}_{n}+t_{n}\left(\bar{v}_{n}, v_{-n}\right) \\
& \geq s(v) \bar{v}_{n}+t_{n}(v)
\end{aligned}
$$

Moreover, by (2), we have

$$
s(v) v_{n}+t_{n}(v) \geq s^{R C}(v) v_{n}+t_{n}^{R C}(v) .
$$

These imply that

$$
s^{R C}\left(\bar{v}_{n}, v_{-n}\right) \bar{v}_{n}+t_{n}^{R C}\left(\bar{v}_{n}, v_{-n}\right)-s(v) \bar{v}_{n} \geq s^{R C}(v) v_{n}+t_{n}^{R C}(v)-s(v) v_{n} .
$$

From Myerson's Lemma and SubClaim 2-5, it holds that

$$
\begin{aligned}
t_{n}^{R C}\left(\bar{v}_{n}, v_{-n}\right) & =t_{n}^{R C}(v)-s^{R C}\left(\bar{v}_{n}, v_{-n}\right) \bar{v}_{n}+s^{R C}(v) v_{n}+\int_{v_{n}}^{\bar{v}_{n}} s^{R C}\left(x_{n}, v_{-n}\right) d x_{n} \\
& =t_{n}^{R C}(v)-s^{R C}\left(\bar{v}_{n}, v_{-n}\right) \bar{v}_{n}+s^{R C}(v) v_{n}+s^{R C}(v)\left(\bar{v}_{n}-v_{n}\right) \\
& =t_{n}^{R C}(v)-s^{R C}\left(\bar{v}_{n}, v_{-n}\right) \bar{v}_{n}+s^{R C}(v) \bar{v}_{n} .
\end{aligned}
$$

Substituting (6) for (5), we have

$$
\left(s^{R C}(v)-s(v)\right)\left(\bar{v}_{n}-v_{n}\right) \geq 0 .
$$

Since, by SubClaim 2-3, $\bar{v}_{n}>v_{n}$, this implies that

$$
s^{R C}(v) \geq s(v) .
$$

By Claim 1, we have the desired result.

Thus, Claim 2 is valid.

Therefore, Case 1 is completed.

Case 2. $\sum_{i=1}^{n} v_{i}<0$.

By an argument similar to Case 1 , we have $s(v)=s^{R C}(v)$. We omit the details.

Case 3. $\sum_{i=1}^{n} v_{i}=0$.

Suppose to the contrary that there exists some $j \in N$ such that

$$
s(v) v_{j}+t_{j}(v)>s^{R C}(v) v_{j}+t_{j}^{R C}(v) .
$$


Then, from (2), it follows that

$$
s(v) \sum_{i \in N} v_{i}+\sum_{i \in N} t_{i}(v)>s^{R C}(v) \sum_{i \in N} v_{i}+\sum_{i \in N} t_{i}^{R C}(v) .
$$

Since $\sum_{i=1}^{n} v_{i}=0$, by $B B$ of $f^{R C}$, we have

$$
\sum_{i \in N} t_{i}(v)>\sum_{i \in N} t_{i}^{R C}(v)=0,
$$

which contradicts the feasibility. Hence, Case 3 is completed.

Therefore, Theorem 1 is valid.

\subsection{Proof of Proposition 1}

Let $f^{*}=\left(s^{*}, t^{*}\right)$ be a strategy-proof mechanism satisfying (i), (ii), and (iii) in Proposition 1. Let $f=(s, t)$ be a strategy-proof mechanism such that for any $v \in V^{n}$ and any $i \in N$,

$$
s(v) v_{i}+t_{i}(v) \geq s^{*}(v) v_{i}+t_{i}^{*}(v) .
$$

We show that for any $v \in V^{n}$ and any $i \in N$,

$$
s(v) v_{i}+t_{i}(v)=s^{*}(v) v_{i}+t_{i}^{*}(v) .
$$

Let $v \in V^{n}$. In the following, we divide the argument into three cases.

Case 1. $\sum_{i=1}^{n} v_{i}>0$.

From Lemma 2, it is sufficient for (8) to show $s(v)=s^{*}(v)$. We show this by the following claims.

Claim 1. $s(v) \geq s^{*}(v)$.

Proof of Claim 1. By the same argument as Claim 1 in proof of Theorem 1, we can show this claim. We omit the details.

Claim 2. $s(v)=s^{*}(v)$.

Proof of Claim 2. We show this claim by the following induction.

1. If $\#\left\{i \in N: v_{i} \geq 0\right\}=n$, then $s(v)=s^{*}(v)$.

2. Let $m$ be such that $1 \leq m \leq n-1$. Assume that for any $v^{\prime} \in V^{n}$ such that $\#\left\{i \in N: \overline{v_{i}^{\prime}} \geq \overline{0\}}=m+1\right.$, we have $s\left(v^{\prime}\right)=s^{*}\left(v^{\prime}\right)$. If $\#\left\{i \in N: v_{i} \geq 0\right\}=m$, then $s(v)=s^{*}(v)$. 
First Part.

Since $\sum_{i=1}^{n} v_{i}>0$, there exists $i \in N$ such that $v_{i}>0$. Since $\#\left\{i \in N: v_{i} \geq\right.$ $0\}=n$, by (ii), we have $s^{*}(v)=1$. Since, by Claim 1 , we have $s(v) \geq s^{*}(v)$, the first part is valid.

Second Part.

Without loss of generality, we assume that $v_{n}<0$. Let $\bar{v}_{n}=0$. Then, by the induction hypothesis, it holds that

$$
s^{*}\left(\bar{v}_{n}, v_{-n}\right)=s\left(\bar{v}_{n}, v_{-n}\right) .
$$

Then, by Lemma 2, it follows that

$$
t^{*}\left(\bar{v}_{n}, v_{-n}\right)=t\left(\bar{v}_{n}, v_{-n}\right) .
$$

Then, by $S P$ of $f$, these imply that

$$
\begin{aligned}
s^{*}\left(\bar{v}_{n}, v_{-n}\right) \bar{v}_{n}+t_{n}^{*}\left(\bar{v}_{n}, v_{-n}\right) & =s\left(\bar{v}_{n}, v_{-n}\right) \bar{v}_{n}+t_{n}\left(\bar{v}_{n}, v_{-n}\right) \\
& \geq s(v) \bar{v}_{n}+t_{n}(v) .
\end{aligned}
$$

Since $\bar{v}_{n}=0$, by (i), it means that

$$
0=t_{n}^{*}\left(\bar{v}_{n}, v_{-n}\right) \geq t_{n}(v) .
$$

Moreover, by (7) and (i), we have

$$
s(v) v_{n}+t_{n}(v) \geq s^{*}(v) v_{n}+t_{n}^{*}(v)=s^{*}(v) v_{n} .
$$

These imply that

$$
0 \geq s^{*}(v) v_{n}-s(v) v_{n}=\left(s^{*}(v)-s(v)\right) v_{n} .
$$

Since $v_{n}<0$, this implies that

$$
s^{*}(v) \geq s(v) .
$$

Since, by Claim 1 , we have $s(v) \geq s^{*}(v)$, the second part is valid. Thus, Claim 2 is valid.

Therefore, Case 1 is completed.

Case 2. $\sum_{i=1}^{n} v_{i}<0$.

By an argument similar to Case 1, we have $s(v)=s^{*}(v)$. We omit the details.

Case 3. $\sum_{i=1}^{n} v_{i}=0$.

By the same argument as Case 3 in proof of Theorem 1, we can show this claim. We omit the details.

Therefore, Proposition 1 is valid. 


\subsection{Proof of Theorem 2}

Note that the welfare loss of the random chair pivotal mechanism at $v$ is

$$
\begin{aligned}
& W L\left(v ; f^{R C}\right)= \max \left\{\sum_{i=1}^{n} v_{i}, 0\right\}-s^{R C}(v) \sum_{i=1}^{n} v_{i} \\
&= \begin{cases}\sum_{i=1}^{n} v_{i}-\frac{1}{n} \#\left\{i \in N: \sum_{k \neq i} v_{k} \geq 0\right\} \sum_{i=1}^{n} v_{i} & \text { if } \sum_{i=1}^{n} v_{i}>0, \\
0-0 & \text { if } \sum_{i=1}^{n} v_{i}=0, \\
0-\frac{1}{n} \#\left\{i \in N: \sum_{k \neq i} v_{k}>0\right\} \sum_{i=1}^{n} v_{i} & \text { if } \sum_{i=1}^{n} v_{i}<0,\end{cases} \\
&= \begin{cases}\frac{1}{n} \#\left\{i \in N: \sum_{k \neq i} v_{k}<0\right\} \sum_{i=1}^{n} v_{i} & \text { if } \sum_{i=1}^{n} v_{i}>0, \\
0 & \text { if } \sum_{i=1}^{n} v_{i}=0, \\
-\frac{1}{n} \#\left\{i \in N: \sum_{k \neq i} v_{k}>0\right\} \sum_{i=1}^{n} v_{i} & \text { if } \sum_{i=1}^{n} v_{i}<0 .\end{cases}
\end{aligned}
$$

Let $\left(v_{i}\right)_{i=1}^{n}$ be a finite sequence of valuations. Let $\left(v_{i}\right)_{i=1}^{n \times r}$ be $r$-replica of $\left(v_{i}\right)_{i=1}^{n}$. When $\sum_{i=1}^{n} v_{i}=0$, since $W L\left(\left(v_{i}\right)_{i=1}^{n \times r} ; f^{R C}\right)=0$, it holds that

$$
\lim _{r \rightarrow \infty} W L\left(\left(v_{i}\right)_{i=1}^{n \times r} ; f^{R C}\right)=0 .
$$

Hence, we consider the case $\sum_{i=1}^{n} v_{i} \neq 0$. Without loss of generality, we assume $\sum_{i=1}^{n} v_{i}>0$. Define $\beta=\max \left\{v_{1}, \ldots, v_{n}\right\}$. Let $\bar{r}$ be such that

$$
\bar{r} \times \sum_{i=1}^{n} v_{i}>\beta .
$$

Then, for any $r>\bar{r}$ and any $i \in\{1, \ldots, n \times r\}$, it holds that

$$
\sum_{k=1}^{n \times r} v_{k}-v_{i}=r \sum_{k=1}^{n} v_{k}-v_{i} \geq r \sum_{k=1}^{n} v_{k}-\beta>0,
$$

that is,

$$
\#\left\{i \in N: \sum_{k=1}^{n \times r} v_{k}-v_{i}<0\right\}=0 .
$$

Hence, we have

$$
\lim _{r \rightarrow \infty} \frac{1}{n \times r} \#\left\{i \in N: \sum_{k=1}^{n \times r} v_{k}-v_{i}<0\right\} \sum_{k=1}^{n \times r} v_{k}=0
$$




\subsection{Proof of Proposition 2}

We exhibit an example of a finite sequence of valuations $\left(v_{i}\right)_{i=1}^{n}$ such that

$$
\lim _{r \rightarrow \infty} W L\left(\left(v_{i}\right)_{i=1}^{n \times r} ; f^{P V}\right)=\infty .
$$

Note that the welfare loss of the pivotal mechanism at $v$ is

$$
W L\left(v ; f^{P V}\right)=-\left(\sum_{i=1}^{n} t_{i}^{P V}(v)\right) .
$$

Let $v_{1}=1$ and $v_{2}=-1$. Let $\left(v_{i}\right)_{i=1}^{2 \times r}$ be $r$-replica of $\left(v_{1}, v_{2}\right)$. Note that for any $r^{\prime}<r$,

$$
t_{2 r^{\prime}+1}^{P V}\left(\left(v_{i}\right)_{i=1}^{2 \times r}\right)+t_{2 r^{\prime}+2}^{P V}\left(\left(v_{i}\right)_{i=1}^{2 \times r}\right)=-1 .
$$

Hence, we have

$$
W L\left(\left(v_{i}\right)_{i=1}^{2 \times r} ; f^{P V}\right)=r .
$$

Thus, $\lim _{r \rightarrow \infty} W L\left(\left(v_{i}\right)_{i=1}^{2 \times r} ; f^{P V}\right)=\infty$.

\subsection{Proof of Proposition 3}

We exhibit an example of a finite sequence of valuations $\left(v_{i}\right)_{i=1}^{n}$ such that

$$
\lim _{r \rightarrow \infty} W L\left(\left(v_{i}\right)_{i=1}^{n \times r} ; f^{M V}\right)=\infty .
$$

Note that the welfare loss of the majority voting mechanism at $v$ is

$$
W L\left(v ; f^{M V}\right)=\max \left\{\sum_{i=1}^{n} v_{i}, 0\right\}-s^{M V}(v) \sum_{i=1}^{n} v_{i} .
$$

Let $v_{1}=3$ and $v_{2}=v_{3}=-1$. Let $\left(v_{i}\right)_{i=1}^{3 \times r}$ be $r$-replica of $\left(v_{1}, v_{2}, v_{3}\right)$. Note that for any $r$,

$$
s^{M V}\left(\left(v_{i}\right)_{i=1}^{3 \times r}\right)=0 .
$$

Hence, we have

$$
W L\left(\left(v_{i}\right)_{i=1}^{3 \times r} ; f^{M V}\right)=r .
$$

Thus, $\lim _{r \rightarrow \infty} W L\left(\left(v_{i}\right)_{i=1}^{3 \times r} ; f^{M V}\right)=\infty$. 


\subsection{Proof of Proposition 4}

We exhibit an example of a finite sequence of valuations $\left(v_{i}\right)_{i=1}^{n}$ such that

$$
\lim _{r \rightarrow \infty} W L\left(\left(v_{i}\right)_{i=1}^{n \times r} ; f^{R D}\right)=\infty .
$$

Note that the welfare loss of the random serial dictatorship mechanism at $v$ is

$$
W L\left(v ; f^{R D}\right)=\max \left\{\sum_{i=1}^{n} v_{i}, 0\right\}-s^{R D}(v) \sum_{i=1}^{n} v_{i} .
$$

Let $v_{1}=2$ and $v_{2}=-1$. Let $\left(v_{i}\right)_{i=1}^{2 \times r}$ be $r$-replica of $\left(v_{1}, v_{2}\right)$. Note that for any $r$,

$$
s^{R D}\left(\left(v_{i}\right)_{i=1}^{2 \times r}\right)=\frac{1}{2} .
$$

Hence, we have

$$
W L\left(\left(v_{i}\right)_{i=1}^{2 \times r} ; f^{R D}\right)=\frac{1}{2} r .
$$

Thus, $\lim _{r \rightarrow \infty} W L\left(\left(v_{i}\right)_{i=1}^{2 \times r} ; f^{R D}\right)=\infty$.

\subsection{Proof of Proposition 5}

We exhibit an example of a finite sequence of valuations $\left(v_{i}\right)_{i=1}^{n}$ such that

$$
\lim _{r \rightarrow \infty} W L\left(\left(v_{i}\right)_{i=1}^{n \times r} ; f^{U N}\right)=\infty .
$$

Note that the welfare loss of the unanimity mechanism at $v$ is

$$
W L\left(v ; f^{U N}\right)=\max \left\{\sum_{i=1}^{n} v_{i}, 0\right\}-s^{U N}(v) \sum_{i=1}^{n} v_{i} .
$$

Let $v_{1}=2$ and $v_{2}=-1$. Let $\left(v_{i}\right)_{i=1}^{2 \times r}$ be $r$-replica of $\left(v_{1}, v_{2}\right)$. Note that for any $r$,

$$
s^{U N}\left(\left(v_{i}\right)_{i=1}^{2 \times r}\right)=\frac{1}{2} .
$$

Hence, we have

$$
W L\left(\left(v_{i}\right)_{i=1}^{2 \times r} ; f^{U N}\right)=\frac{1}{2} r .
$$

Thus, $\lim _{r \rightarrow \infty} W L\left(\left(v_{i}\right)_{i=1}^{2 \times r} ; f^{U N}\right)=\infty$. 


\subsection{Proof of Theorem 3}

It is obvious that the random chair pivotal mechanism satisfies the axioms. Hence, we omit the "if" part.

In the following, we show the "only if" part. Let $f=(s, t)$ be a mechanism satisfying strategy-proofness, budget-balance, equal treatment of equals, and decision-robustness.

Claim 1. For any $v \in V^{n}$ such that $\sum_{i \in N} v_{i}>0$, we have $f(v)=f^{R C}(v)$.

Proof of Claim 1. We first show $s(v)=s^{R C}(v)$. Next, we show $t(v)=$ $t^{R C}(v)$.

SubClaim 1-1. For any $v \in V^{n}$ such that $\sum_{i \in N} v_{i}>0$, we have $s(v)=$ $s^{R C}(v)$.

Proof of SubClaim 1-1. Let $v \in V^{n}$ be such that $\sum_{i \in N} v_{i}>0$. By DR, we have

$$
s(v)=\frac{1}{n} \#\left\{i \in N: \sum_{k \neq i} v_{k} \geq 0\right\} .
$$

By definition, for any $i \in N$ such that $\sum_{k \neq i} v_{k}>0$, we have $d^{-i}(v)=1$. Similarly, for any $i \in N$ such that $\sum_{k \neq i} v_{k}<0$, we have $d^{-i}(v)=0$. Since $\sum_{i \in N} v_{i}>0, \sum_{k \neq i} v_{k}=0$ means $v_{i}>0$. Hence, for any $i \in N$ such that $\sum_{k \neq i} v_{k}=0$, we have $d^{-i}(v)=1$. Thus, it follows that

$$
\#\left\{i \in N: \sum_{k \neq i} v_{k} \geq 0\right\}=\sum_{i \in N} d^{-i}(v)
$$

which implies that

$$
s(v)=s^{R C}(v)
$$

SubClaim 1-2. For any $v \in V^{n}$ such that $\sum_{i \in N} v_{i}>0$, we have $t(v)=$ $t^{R C}(v)$.

Proof of SubClaim 1-2. Let $v \in V^{n}$ be such that $\sum_{i \in N} v_{i}>0$. We show this SubClaim by the following induction.

1. If $s^{R C}(v)=1$, then $t(v)=t^{R C}(v)$.

2. Let $m$ be such that $1 \leq m \leq n-1$. Assume that for any $v^{\prime} \in V^{n}$ such that $\sum_{i \in N} v_{i}^{\prime}>0$ and $s^{R C}\left(v^{\prime}\right)>\frac{n-m}{n}$, we have $t\left(v^{\prime}\right)=t^{R C}\left(v^{\prime}\right)$. If $s^{R C}(v)=\frac{n-m}{n}$, then $t(v)=t^{R C}(v)$. 
Without loss of generality, we assume that $v_{1} \geq v_{2} \geq \cdots \geq v_{n}$.

First Part.

Assume $s^{R C}(v)=1$. Suppose to the contrary that there exists $j \in N$ such that $t_{j}(v) \neq t_{j}^{R C}(v)$. By $B B$, we can take $j \neq 1$. Notice that for any $\hat{v} \in V^{n}$ such that $s^{R C}(\hat{v})=1$ and any $i \in N$, it holds that $t_{i}^{R C}(\hat{v})=0$. That is, $t_{j}(v) \neq 0$. Let $\bar{v}_{j}=v_{1}$. Since $\bar{v}_{j} \geq v_{j}$, from Myerson's Lemma, we have

$$
s\left(\bar{v}_{j}, v_{-j}\right) \geq s(v)=s^{R C}(v)=1,
$$

that is,

$$
s\left(\bar{v}_{j}, v_{-j}\right)=s(v)
$$

Then, by $S P$, we also have

$$
t_{j}\left(\bar{v}_{j}, v_{-j}\right)=t_{j}(v)
$$

By ETE, we have

$$
t_{1}\left(\bar{v}_{j}, v_{-j}\right)=t_{j}\left(\bar{v}_{j}, v_{-j}\right) \neq 0 .
$$

Then, by $B B$, there exists $k \neq 1, j$ such that $t_{k}\left(\bar{v}_{j}, v_{-j}\right) \neq 0$. Let $\bar{v}_{k}=v_{1}$. Then, by the same argument, we have

$$
t_{1}\left(\bar{v}_{j}, \bar{v}_{k}, v_{-j k}\right)=t_{j}\left(\bar{v}_{j}, \bar{v}_{k}, v_{-j k}\right)=t_{k}\left(\bar{v}_{j}, \bar{v}_{k}, v_{-j k}\right) \neq 0 .
$$

Repeating the same argument, it follows that for any $i \in N$,

$$
t_{1}\left(v_{1}, \bar{v}_{2}, \ldots, \bar{v}_{n}\right)=t_{i}\left(v_{1}, \bar{v}_{2}, \ldots, \bar{v}_{n}\right) \neq 0
$$

which contradicts $B B$. Thus, the first part is valid.

Second Part.

Assume $s^{R C}(v)=\frac{n-m}{n}$. Let $k \neq 1$. Let $v_{k}^{\prime} \in V$ be such that $v_{k}^{\prime}>v_{k}$. By taking a sufficiently large $v_{k}^{\prime}$, we have $s^{R C}\left(v_{k}^{\prime}, v_{-k}\right)>\frac{n-m}{n}$. Then, by induction hypothesis, we have

$$
t_{k}\left(v_{k}^{\prime}, v_{-k}\right)=t_{k}^{R C}\left(v_{k}^{\prime}, v_{-k}\right) .
$$

Then, from Myerson's Lemma, it follows that

$$
\begin{aligned}
t_{k}\left(v_{k}, v_{-k}\right) & -s\left(v_{k}^{\prime}, v_{-k}\right) v_{k}^{\prime}+s\left(v_{k}, v_{-k}\right) v_{k}+\int_{v_{k}}^{v_{k}^{\prime}} s\left(x_{k}, v_{-k}\right) d x_{k} \\
& =t_{k}\left(v_{k}^{\prime}, v_{-k}\right) \\
& =t_{k}^{R C}\left(v_{k}^{\prime}, v_{-k}\right) \\
& =t_{k}^{R C}\left(v_{k}, v_{-k}\right)-s^{R C}\left(v_{k}^{\prime}, v_{-k}\right) v_{k}^{\prime}+s^{R C}\left(v_{k}, v_{-k}\right) v_{k}+\int_{v_{k}}^{v_{k}^{\prime}} s^{R C}\left(x_{k}, v_{-k}\right) d x_{k} .
\end{aligned}
$$


Since $\sum_{i \in N} v_{i}>0$, for any $x_{k} \geq v_{k}$, we have $\sum_{i \neq k} v_{i}+x_{k}>0$, which, by SubClaim 1-1, implies $s\left(x_{k}, v_{-k}\right)=s^{R C}\left(x_{k}, v_{-k}\right)$. Hence, we have

$$
t_{k}\left(v_{k}, v_{-k}\right)=t_{k}^{R C}\left(v_{k}, v_{-k}\right) .
$$

Then, by $B B$, we also have

$$
t_{1}(v)=t_{1}^{R C}(v)
$$

Thus, the second part is valid. Hence, SubClaim 1-2 is valid.

Therefore, Claim 1 is valid.

Claim 2. For any $v \in V^{n}$ such that $\sum_{i \in N} v_{i}<0$, we have $f(v)=f^{R C}(v)$.

Proof of Claim 2. By an argument similar to Claim 1, we can show Claim 2. We omit the details.

Claim 3. For any $v \in V^{n}$ such that $\sum_{i \in N} v_{i}=0$ and that for any $i \in N$, $v_{i} \neq 0$, we have $f(v)=f^{R C}(v)$.

Proof of Claim 3. We first show $s(v)=s^{R C}(v)$. Next, we show $t(v)=$ $t^{R C}(v)$.

SubClaim 3-1. For any $v \in V^{n}$ such that $\sum_{i \in N} v_{i}=0$ and that for any $i \in N, v_{i} \neq 0$, we have $s(v)=s^{R C}(v)$.

Proof of SubClaim 3-1. Let $v \in V^{n}$ be such that $\sum_{i \in N} v_{i}=0$ and that for any $i \in N, v_{i} \neq 0$. Note that for any $i \in N, \sum_{k \neq i} v_{k} \neq 0$. Then, by taking $\bar{v}_{1}>v_{1}$ sufficiently close to $v_{1}$, we can have that for any $i \in N$,

$$
d^{-i}(v)=d^{-i}\left(\bar{v}_{1}, v_{-1}\right),
$$

that is,

$$
s^{R C}(v)=s^{R C}\left(\bar{v}_{1}, v_{-1}\right) .
$$

Since $\sum_{k \neq 1} v_{k}+\bar{v}_{1}>0$, by Claim 1 , it holds that

$$
s\left(\bar{v}_{1}, v_{-1}\right)=s^{R C}\left(\bar{v}_{1}, v_{-1}\right) .
$$

Similarly, by taking $\hat{v}_{1}<v_{1}$ sufficiently close to $v_{1}$, we can have that for any $i \in N$,

$$
d^{-i}(v)=d^{-i}\left(\hat{v}_{1}, v_{-1}\right)
$$

that is,

$$
s^{R C}(v)=s^{R C}\left(\hat{v}_{1}, v_{-1}\right) .
$$


Since $\sum_{k \neq 1} v_{k}+\hat{v}_{1}<0$, by Claim 2, it holds that

$$
s\left(\hat{v}_{1}, v_{-1}\right)=s^{R C}\left(\hat{v}_{1}, v_{-1}\right) .
$$

Then, from Myerson's Lemma, these imply that

$$
s^{R C}(v)=s\left(\hat{v}_{1}, v_{-1}\right) \leq s(v) \leq s\left(\bar{v}_{1}, v_{-1}\right)=s^{R C}(v),
$$

which means the desired result.

SubClaim 3-2. For any $v \in V^{n}$ such that $\sum_{i \in N} v_{i}=0$ and that for any $i \in N, v_{i} \neq 0$, we have $t(v)=t^{R C}(v)$.

Proof of SubClaim 3-2. Let $v \in V^{n}$ be such that $\sum_{i \in N} v_{i}=0$ and that for any $i \in N, v_{i} \neq 0$. Let $k \in N$. Let $v_{k}^{\prime}>v_{k}$. Then, for any $x_{k} \in V$ such that $v_{k}<x_{k} \leq v_{k}^{\prime}$, since $\sum_{i \neq k} v_{i}+x_{k}>0$, by Claim 1, we have $f\left(x_{k}, v_{-k}\right)=f^{R C}\left(x_{k}, v_{-k}\right)$. Then, from Myerson's Lemma, it follows that

$$
\begin{aligned}
t_{k}\left(v_{k}, v_{-k}\right) & -s\left(v_{k}^{\prime}, v_{-k}\right) v_{k}^{\prime}+s\left(v_{k}, v_{-k}\right) v_{k}+\int_{v_{k}}^{v_{k}^{\prime}} s\left(x_{k}, v_{-k}\right) d x_{k} \\
& =t_{k}\left(v_{k}^{\prime}, v_{-k}\right) \\
& =t_{k}^{R C}\left(v_{k}^{\prime}, v_{-k}\right) \\
& =t_{k}^{R C}\left(v_{k}, v_{-k}\right)-s^{R C}\left(v_{k}^{\prime}, v_{-k}\right) v_{k}^{\prime}+s^{R C}\left(v_{k}, v_{-k}\right) v_{k}+\int_{v_{k}}^{v_{k}^{\prime}} s^{R C}\left(x_{k}, v_{-k}\right) d x_{k} .
\end{aligned}
$$

Since, by SubClaim 3-1, $s\left(v_{k}, v_{-k}\right)=s^{R C}\left(v_{k}, v_{-k}\right)$, this implies that

$$
t_{k}\left(v_{k}, v_{-k}\right)=t_{k}^{R C}\left(v_{k}, v_{-k}\right) .
$$

Thus, SubClaim 3-2 is valid.

Therefore, Claim 3 is valid.

Claim 4. For any $v \in V^{n}$ such that $\sum_{i \in N} v_{i}=0$ and that for some $i \in N$, $v_{i}=0$, we have $f(v)=f^{R C}(v)$.

Proof of Claim 4. We first show $s(v)=s^{R C}(v)$ by constructing ${ }^{15}$ an arbitrary value $y \in[0,1]$. Next, we show $t(v)=t^{R C}(v)$.

SubClaim 4-1. For any $v \in V^{n}$ such that $\sum_{i \in N} v_{i}=0$ and that for some $i \in N, v_{i}=0$, we have $s(v)=s^{R C}(v)$.

\footnotetext{
${ }^{15}$ See the definition of $d^{-i}$.
} 
Proof of SubClaim 4-1. Let $v \in V^{n}$ be such that $\sum_{i \in N} v_{i}=0$ and that for some $i \in N, v_{i}=0$. Let $N^{0}=\left\{i \in N: v_{i}=0\right\}$ and $\# N^{0}=n^{0}$. Let $N^{-}=\left\{i \in N: v_{i}<0\right\}$ and $\# N^{-}=n^{-}$.

Define $y=\frac{n}{n^{0}}\left(s(v)-\frac{1}{n} n^{-}\right)$. We show that $0 \leq y \leq 1$. Note that for any $i \in N \backslash N^{0}, \sum_{k \neq i} v_{k} \neq 0$. Hence, by taking $\hat{v}_{1}<v_{1}$ sufficiently close to $v_{1}$, we can have that for any $i \in N \backslash N^{0}$,

$$
d^{-i}(v)=d^{-i}\left(\hat{v}_{1}, v_{-1}\right),
$$

that is,

$$
\sum_{i \in N \backslash N^{0}} d^{-i}(v)=\sum_{i \in N \backslash N^{0}} d^{-i}\left(\hat{v}_{1}, v_{-1}\right) .
$$

Note that

$$
\sum_{i \in N \backslash N^{0}} d^{-i}(v)=n^{-} .
$$

Note also that for any $i \in N^{0}$, it holds that

$$
d^{-i}\left(\hat{v}_{1}, v_{-1}\right)=0 .
$$

Then, since $\sum_{i \neq 1} v_{i}+\hat{v}_{1}<0$, by Claim 2, we have

$$
s\left(\hat{v}_{1}, v_{-1}\right)=s^{R C}\left(\hat{v}_{1}, v_{-1}\right) \equiv \frac{1}{n} \sum_{i \in N} d^{-i}\left(\hat{v}_{1}, v_{-1}\right)=\frac{1}{n} n^{-} .
$$

Since $\hat{v}_{1}<v_{1}$, from Myerson's Lemma, we have

$$
s(v) \geq s\left(\hat{v}_{1}, v_{-1}\right)=\frac{1}{n} n^{-} .
$$

Hence, $y \geq 0$. Similarly, by taking $\bar{v}_{1}>v_{1}$ sufficiently close to $v_{1}$, we can have that for any $i \in N \backslash N^{0}$,

$$
d^{-i}(v)=d^{-i}\left(\bar{v}_{1}, v_{-1}\right),
$$

that is,

$$
n^{-}=\sum_{i \in N \backslash N^{0}} d^{-i}(v)=\sum_{i \in N \backslash N^{0}} d^{-i}\left(\bar{v}_{1}, v_{-1}\right) .
$$

Note that for any $i \in N^{0}$, it holds that

$$
d^{-i}\left(\bar{v}_{1}, v_{-1}\right)=1 .
$$

Then, since $\sum_{i \neq 1} v_{i}+\bar{v}_{1}>0$, by Claim 1 , we have

$$
s\left(\bar{v}_{1}, v_{-1}\right)=s^{R C}\left(\bar{v}_{1}, v_{-1}\right) \equiv \frac{1}{n} \sum_{i \in N} d^{-i}\left(\bar{v}_{1}, v_{-1}\right)=\frac{1}{n}\left(n^{0}+n^{-}\right) .
$$


Since $\bar{v}_{1}>v_{1}$, from Myerson's Lemma, we have

$$
s(v) \leq s\left(\bar{v}_{1}, v_{-1}\right)=\frac{1}{n}\left(n^{0}+n^{-}\right) .
$$

Hence, $y \leq 1$.

For any $i \in N^{0}$, set $d^{-i}(v)=y$. Then, it follows that

$$
\begin{aligned}
s^{R C}(v) & \equiv \frac{1}{n} \sum_{i \in N} d^{-i}(v) \\
& =\frac{1}{n} \sum_{i \in N^{0}} d^{-i}(v)+\frac{1}{n} \sum_{i \in N \backslash N^{0}} d^{-i}(v) \\
& =\frac{1}{n} n^{0} y+\frac{1}{n} n^{-} \\
& =s(v)-\frac{1}{n} n^{-}+\frac{1}{n} n^{-}=s(v) .
\end{aligned}
$$

Thus, SubClaim 4-1 is valid.

SubClaim 4-2. For any $v \in V^{n}$ such that $\sum_{i \in N} v_{i}=0$ and that for some $i \in N, v_{i}=0$, we have $t(v)=t^{R C}(v)$.

Proof of SubClaim 4-2. By an argument similar to SubClaim 3-2, we can prove SubClaim 4-2. We omit the details.

Thus, Claim 4 is valid.

Therefore, Theorem 3 is valid.

Acknowledgments The authors thank Tomoya Kazumura, Shuhei Morimoto, Yu Nakayama, Shigehiro Serizawa, and Ken Urai for their helpful comments. This work was supported by JSPS KAKENHI Grant Number 26780117.

\section{References}

Bailey, M.J., 1997, The Demand Revealing Process: To Distribute the Surplus, Public Choice 91, 107-126.

Carbajal, J.C., A. McLennan, T. Tourky, 2013, Truthful Implementation and Preference Aggregation in Restricted Domains, Journal of Economic Theory 148, 1074-1101. 
Clarke, E., 1971, Multipart Pricing of Public Goods, Public Choice 11, 17-33.

Faltings, B., 2005, A Budget-Balanced, Incentive-Compatible Scheme for Social Choice, In Peyman Faratin and Juan A. Rodriguez-Aguilar, editors, Agent-Mediated Electronic Commerce VI 3435, 30-43.

Green, J., E. Kohlberg, J.J. Laffont, 1976, Partial Equilibrium Approach to the Free-Rider Problem, Journal of Public Economics 6, 375-394.

Green, J., J.J. Laffont, 1977, Characterization of Satisfactory Mechanisms for the Revelation of Preferences for Public Goods, Econometrica 45, 427-438.

Green, J., J.J. Laffont, 1979, Incentives in Public Decision Making (Studies in Public Economics), North-Holland.

Groves, T., 1973, Incentives in Teams, Econometrica 41, 617-631.

Groves, T., J.O. Ledyard, 1977, Some Limitations of Demand Revealing Processes, Public Choice 29, 108-124.

Groves, T., M. Loeb, 1975, Incentives and Public Inputs, Journal of Public Economics 4, 211-226.

Guo, M., V. Naroditskiy, V. Conitzer, A. Greenwald, N.R. Jennings, 2011, Budget-Balanced and Nearly Efficient Randomized Mechanisms: Public Goods and Beyond, Proceedings of the Workshop on Internet and Network Economics, 158-169.

Holmström, B., 1979, Groves Scheme on Restricted Domain, Econometrica 47, 1137-1144.

Laffont, J.J., E. Maskin, 1980, A Differential Approach to Dominant Strategy Mechanisms, Econometrica 48, 1507-1520.

Liu, L., G. Tian, 1999, A Characterization of the Existence of Optimal Dominant Strategy Mechanisms, Review of Economic Design 4, 205-218.

Marchant, T., D. Mishra, 2015, Mechanism Design with Two Alternatives in Quasi-linear Environments, Social Choice and Welfare 44, 433-455.

Mishra, D., A. Sen, 2012, Roberts' Theorem with Neutrality: A Social Welfare Ordering Approach, Games and Economic Behavior 75, 283-298.

Mitsui, T., 1983, Asymptotic Efficiency of the Pivotal Mechanism with General Project Space, Journal of Economic Theory 31, 318-331. 
Moulin, H., 1986, Characterizations of the Pivotal Mechanism, Journal of Public Economics 31, 53-78.

Moulin, H., 1994, Serial Cost-Sharing of Excludable Public Goods, Review of Economic Studies 61, 305-325.

Myerson, R.B., 1981, Optimal Auction Design. Mathematics of Operations Research 6, 58-73.

Ohseto, S., 2000, Characterization of Strategy-Proof Mechanisms for Excludable versus Nonexcludable Public Projects, Games and Economic Behavior 32, 51-66.

Rob, R., 1982, Asymptotic Efficiency of the Demand Revealing Mechanism, Journal of Economic Theory 28, 207-220.

Serizawa, S., 1996, Strategy-Proof and Individually Rational Social Choice Functions for Public Good Economies, Economic Theory 7, 501-512.

Serizawa, S., 1999, Strategy-Proof and Symmetric Social Choice Functions for Public Good Economies, Econometrica 67, 121-145.

Tian, G., 1996, On the Existence of Optimal Truth-Dominant Mechanisms, Economics Letters 53, 17-24.

Vickrey, W., 1961, Counterspeculation, Auctions, and Competitive Sealed Tenders. Journal of Finance 16, 8-37.

Zhou, L., 2007, The Failure of Groves Mechanisms in Canonical Allocation Models. mimeo. 


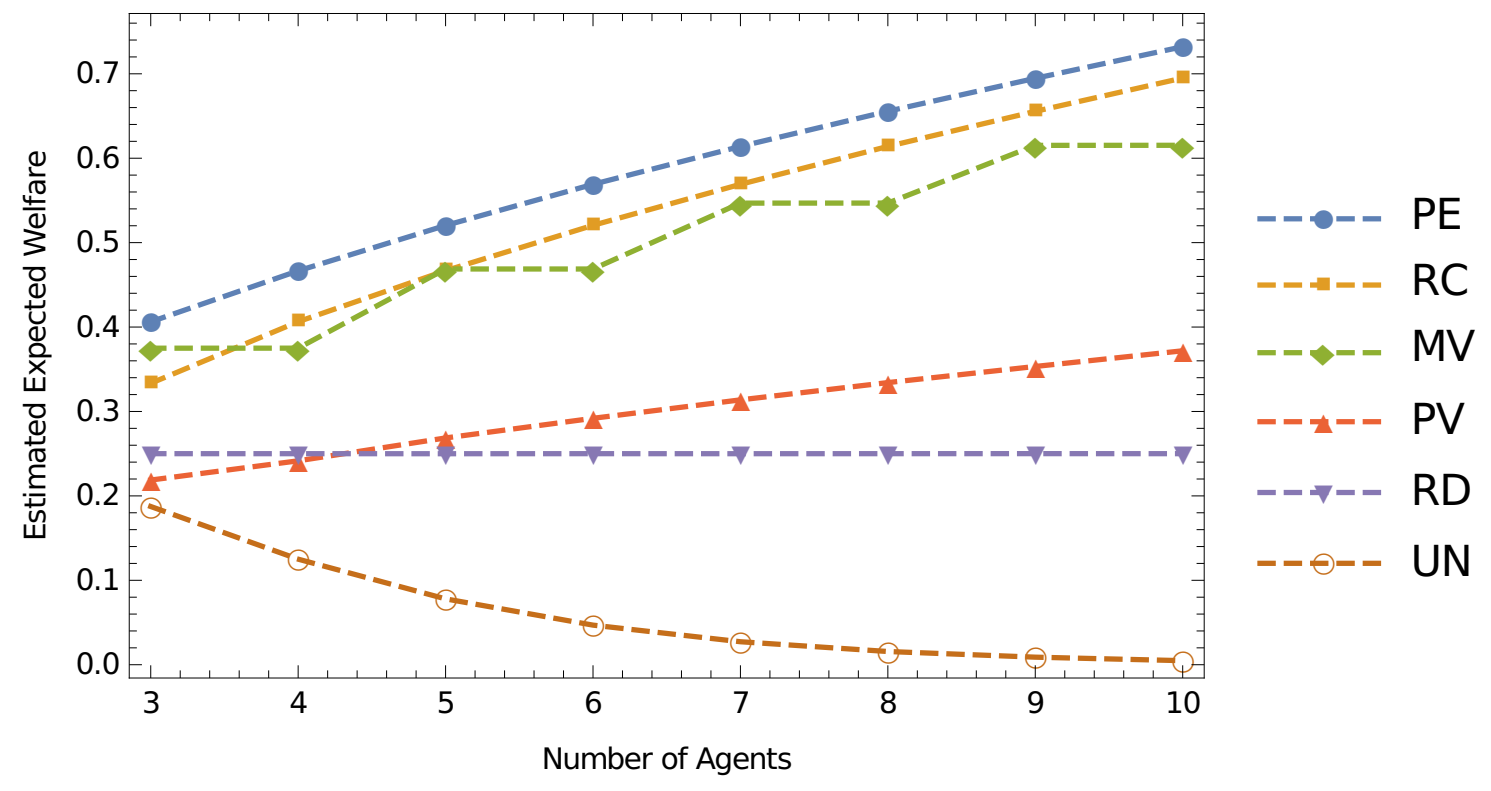

Figure 1: Expected Welfare on Uniform Distribution.

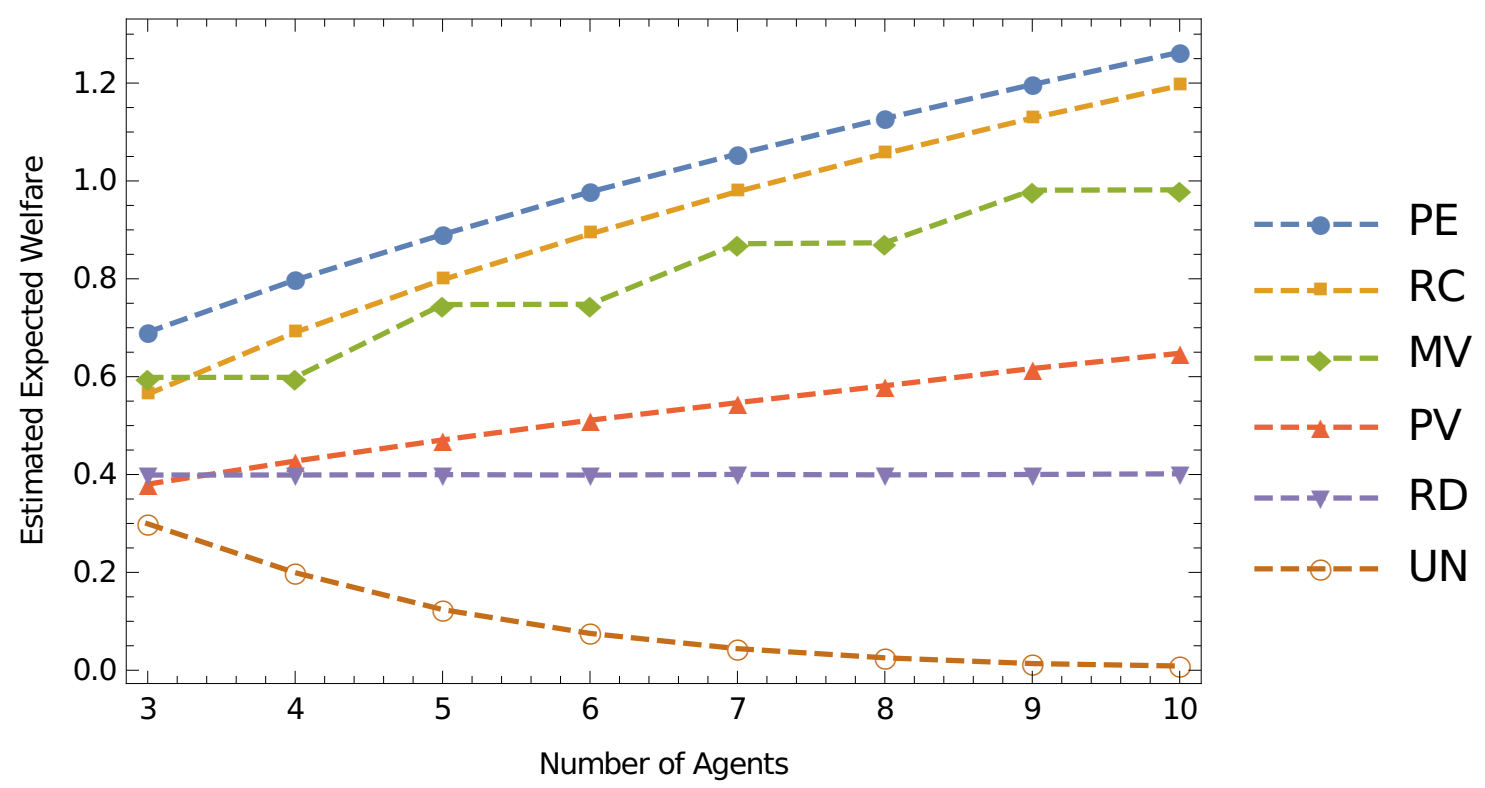

Figure 2: Expected Welfare on Normal Distribution. 